\title{
'Rupture' Type Thrombopoiesis from Bone Megakaryocyte is Regulated by IL- 1Alpha: Visualization by Two Photon Microscopy and Software Analysis
}

\author{
Satoshi Nishimura ${ }^{1,2,3}$ Akira Sawaguchi $^{4}$ \\ 1. Center for Molecular Medicine, Jichi Medical University, Tochigi, Japan \\ 2. Department of Cardiovascular Medicine, the University of Tokyo, Japan \\ 3. Translational Systems Biology and Medicine Initiative, the University of Tokyo, Japan \\ 4. Dept of Anatomy, Ultrastructural Cell Biology, Faculty of Medicine, University of Miyazaki, Japan \\ snishi-tky@umin.ac.jp
}

Blood platelets are generated in the bone marrow (BM) from their precursors, megakaryocytes (MK). Although we know that MKs produced many platelets throughout life, precisely how platelets are produced and how circulating numbers are tightly regulated in vivo remain uncertain, largely because of the rarity of MKs in the BM and the lack an adequate visualization technique. Thus, we utilized highspeed XYZT two photon imaging using GaAs detectors, and rapid resonance scanning mirrors for intravital visualization of BM MKs. This system enabled us to evaluate the detailed thrombopoietic processes from BM MKs.

By visualizing living bone marrow in vivo, we identified that a second thrombopoietic process, 'rupture'-like MK fragmentation, can be ongoing simultaneously with previously identified 'proplatelet formation' in the same mouse BM. Rupture was identified as megakaryocyte death with small plateletlike particles into vessels. We defined these two types by quantifying three dimensional deformation and perimeter changes (Fig). It was also revealed that the 'rupture' type produced 40 platelets from one MK in one minute, which was larger than that by proplatelet thrombopoiesis (only 2 from one MK). Large number platelet release processes in rupturing MKs take only few minutes, and easily escaped from conventional morphological evaluation methods.

Careful examinations using image analysis software elucidated that platelets are released preferentially into vessel lumens during 'rupture' type thrombopoiesis.

Proplatelets were dominant thrombopoietic processes under steady state, which was regulated by thrombopoietin (TPO). Conversely, following blood loss, 5-FU administration, antibody-based platelet depletion or acute inflammation, there was accelerated release of large number platelets from MKs mediated by novel 'rupture' type MK behaviors. Rupture was regulated by the interleukin-1 (IL-1)alphatype1 IL-1 receptor axis and ERK-dependent MK atypical apoptosis. It is known that proper microtubule assembly is vital for proplatelet formations in TPO-stimulated MKs, but did not take place in IL-1alpha-stimulated MKs due to uncoordinated expression tubulin. We also revealed the functional instability of plasma membrane after IL-1alpha treatment in MKs using FRAP analysis. AFM showed that IL-1alpha treated MK showed decreased "stiffness" and "contractile force", which reflect the tendency to rupture type thrombopoiesis.

These findings support the ideas that IL-1alpha acts acutely as a platelet releasing factor, coordinating with TPO to dynamically modulate the cellular programming of MKs that regulates platelet counts. 

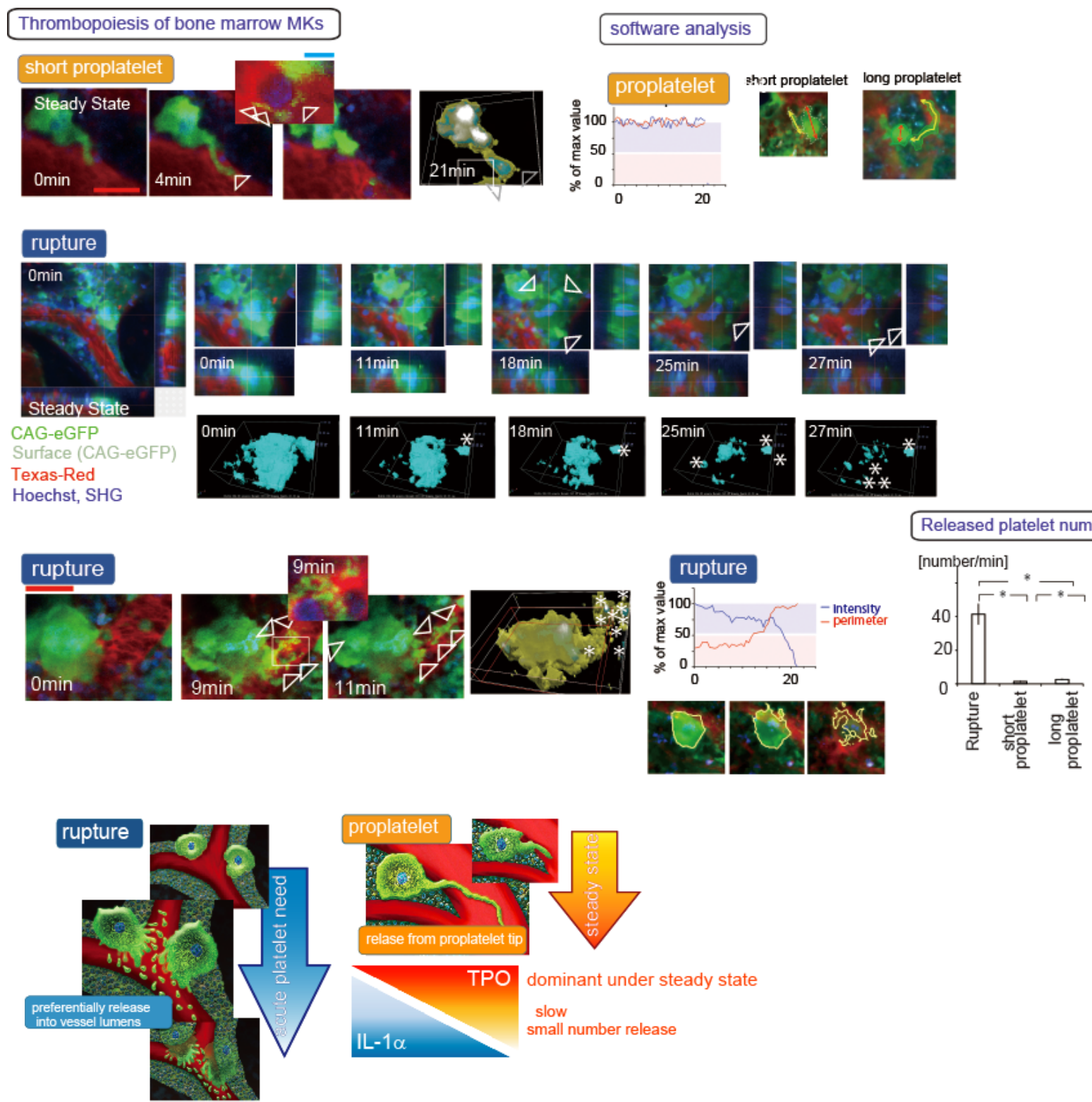

in response to acute platelet need

rapid time course

large number release

MK death, Casp activation

Figure 1

References:

[1] Nishimura S, et al. Cell Metabolism. 2013, 18, 759-766

[2] Nakamura S, Takayama N, „,, Nishimura S, Eto K Cell Stem Cell, 2014 in publication.

[3] Nishimura S, et al. Blood. 2012 ;119(8):e45-56.

[4] Takayama N, Nishimura S, et al. J Exp Med, 2010; 207(13):2817-2830

[5] Takizawa H*, Nishimura S*, et al. (*equal contribution) J Clin Invest., 2010, 120(1): 179-190.

[6] Nishimura S, et al. Nature Medicine, 2009, 15:8, 914-920.

[7] Nishimura S, S. et al. Progress in Biophysics and Molecular Biology, 2008;97:282-297.

[8] Nishimura S, et al. J Clin Invest. 2008, 118(2): 710-721.

[9] Nishimura S, et al. Diabetes. 2007,56:1517-1526. 